\title{
Direct detection of neutralino dark matter with DM@NLO
}

\author{
Michael Klasen* ${ }^{\dagger}$ \\ Institut für Theoretische Physik, Westfälische Wilhelms-Universität Münster, \\ Wilhelm-Klemm-Straße 9, D-48149 Münster, Germany \\ E-mail: michael.klasen@uni-muenster.de
}

\section{Karol Kovařík}

Institut für Theoretische Physik, Westfälische Wilhelms-Universität Münster, Wilhelm-Klemm-Straße 9, D-48149 Münster, Germany

E-mail: karol.kovarikeuni-muenster.de

\section{Saskia Schmiemann}

Institut für Theoretische Physik, Westfälische Wilhelms-Universität Münster, Wilhelm-Klemm-Straße 9, D-48149 Münster, Germany

E-mail: saskia.schmiemanneuni-muenster.de

We calculate spin-independent and spin-dependent direct detection cross sections of neutralino dark matter at next-to-leading order of QCD. The numerical effects are comparable in size to the uncertainties in the nuclear matrix elements. Our results are applicable to bino, wino or higgsino dark matter and allow for consistent correlations with the relic density in DM@NLO.

EPS-HEP 2017, European Physical Society conference on High Energy Physics

5-12 July 2017

Venice, Italy

\footnotetext{
${ }^{*}$ Speaker.

${ }^{\dagger}$ Supported by BMBF under contract 05H15PMCCA.

${ }^{\star}$ Supported by DFG under contract GRK 2149.
} 


\section{Direct detection of neutralino dark matter}

The identification of dark matter (DM) is one of the most urgent questions in astroparticle physics. For many decades, evidence for its sizeable presence in the Universe and its important role in structure formation has been accumulating, and the overall relic density $\Omega h^{2}$ ( $h$ being the present Hubble expansion rate in units of $100 \mathrm{~km} \mathrm{~s}^{-1} \mathrm{Mpc}^{-1}$ ) has been precisely measured [1]. The lightest neutral supersymmetric (SUSY) partner of electroweak gauge and Higgs bosons (neutralino $\tilde{\chi}_{1}^{0}$ ) continues to be a prime candidate for WIMP (weakly interacting massive particle) DM, and theoretical calculations of the DM relic density at next-to-leading order (NLO) of QCD with DM@NLO, which include all coannihilation channels (with the exception of $\tilde{t}_{1} \tilde{t}_{1} \rightarrow q \bar{q}, g g$ ) now match the experimental precision [2]. For an unambiguous identification of DM, it must, however, be detected on Earth, e.g. with large kryogenic detectors like XENON1T [3]. Comparisons with theoretical cross section calculations and correlations with the relic density or other observables (e.g. from indirect detection or the LHC) should then allow for a precise extraction of the DM mass and couplings. For neutralinos, this is now possible thanks to the calculation of NLO SUSY-QCD corrections to the neutralino-nucleon cross section and the inclusion of this second DM observable in DM@NLO [4].

\section{Neutralino-nucleon cross section}

The differential rate for direct DM detection (in counts $/ \mathrm{kg} / \mathrm{day} / \mathrm{keV}$ )

$$
\frac{\mathrm{d} R}{\mathrm{~d} E}=\sum_{i} c_{i} \frac{\sigma_{i}}{2 m_{\tilde{\chi}_{1}^{0}} \mu_{i}^{2}} \rho_{0} \eta_{i}
$$

is usually expressed in terms of the nuclear mass fractions $c_{i}$, reduced masses $\mu_{i}$, local DM density $\rho_{0}=0.3 \mathrm{GeV} / \mathrm{cm}^{3}$, and velocity integrals $\eta_{i}=\int_{v_{\min , i}}^{v_{\text {esc }}} \mathrm{d}^{3} v f(\vec{v}) / v$ with $v_{\min , i}=\sqrt{m_{i} E /\left(2 \mu_{i}^{2}\right)}$.

Since the spin-independent cross sections for each isotope in the target

$$
\sigma_{i}^{\mathrm{SI}}=\frac{\mu_{i}^{2}}{\pi}\left|Z_{i} g_{p}^{\mathrm{SI}}+\left(A_{i}-Z_{i}\right) g_{n}^{\mathrm{SI}}\right|^{2}\left|F_{i}^{\mathrm{SI}}\left(Q_{i}\right)\right|^{2}
$$

depend on the nuclear charges $Z_{i}$, masses $A_{i}$ and structure functions $F_{i}^{\mathrm{SI}}$, they are often replaced by the one for a single nucleon (assuming $g_{p}=g_{n}$ ) to enable a direct comparison of different experiments. We use, however, the exact expressions

$$
g_{N}^{\mathrm{SI}}=\sum_{q}\langle N|\bar{q} q| N\rangle \alpha_{q}^{\mathrm{SI}}
$$

for the spin-independent four-fermion couplings. The Wilson coefficients $\alpha_{q}^{\mathrm{SI}}$ contain the wanted information on the electroweak interaction of DM and quarks, while the nuclear matrix elements $\left\langle N\left|m_{q} \bar{q} q\right| N\right\rangle=f_{T q}^{N} m_{N}$ are known to be subject to considerable uncertainties from the non-perturbative regime of QCD [5, 6, 7]. Beyond the tree-level, the Wilson coefficients $\alpha_{q}^{\mathrm{SI}}$ are, however, also affected by (perturbative) QCD uncertainties and become related to the nuclear matrix elements through renormalisation group equations. ${ }^{1}$

\footnotetext{
${ }^{1}$ The role of effective gluon interactions has been discussed in Ref. [8].
} 
Similarly, the spin-dependent cross section

$$
\sigma_{i}^{\mathrm{SD}}=\frac{4 \mu_{i}^{2}}{2 J+1}\left(\left|g_{p}^{\mathrm{SD}}\right|^{2} S_{\mathrm{pp}, i}\left(Q_{i}\right)+\left|g_{n}^{\mathrm{SD}}\right|^{2} S_{\mathrm{nn}, i}\left(Q_{i}\right)+\left|g_{p}^{\mathrm{SD}} g_{n}^{\mathrm{SD}}\right| S_{\mathrm{pn}, i}\left(Q_{i}\right)\right)
$$

depends on the spin structure functions $S_{N N, i}$ and spin-dependent four-fermion couplings

$$
g_{N}^{\mathrm{SD}}=\sum_{q=u, d, s}(\Delta q)_{N} \alpha_{q}^{\mathrm{SD}} .
$$

Here, the nuclear spin $J$ is supposed to be carried mostly by the three light quark flavours and to be isospin symmetric. ${ }^{2}$

The tree-level diagrams for neutralino-quark scattering are shown in Fig. 1. After the calcula-

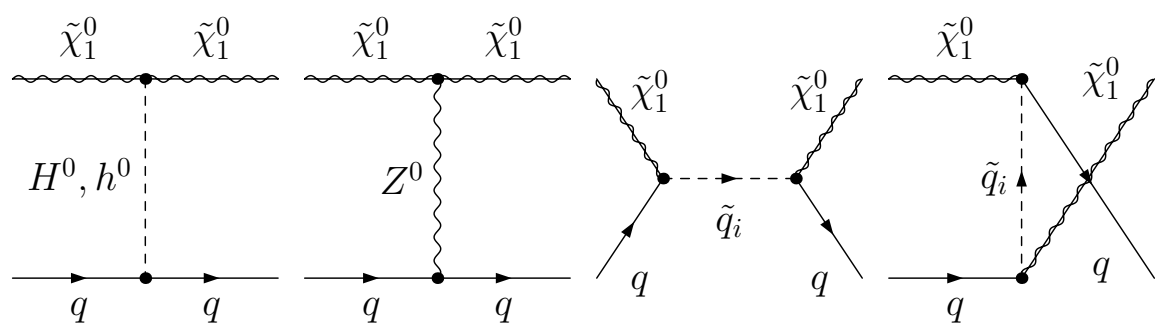

Figure 1: Full tree-level Feynman diagrams for neutralino-quark scattering.

tion of all self-energy, vertex and box corrections, we renormalise the ultraviolet (UV) divergences in a mixed on-shell and $\overline{\mathrm{DR}}$ scheme [4]. It has the advantages of being perturbatively stable, in particular in the top sector, and of allowing for meaningful correlations with our relic density calculations [2] and tree-level comparisons with micrOMEGAs [6], where the same on-shell squark masses are used that are provided by the SUSY spectrum generator SPheno [11].

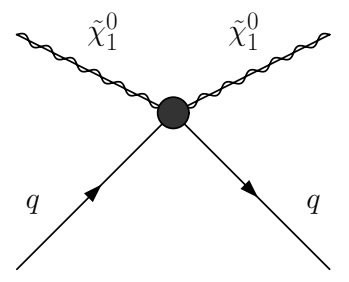

Figure 2: Effective tree-level diagram for neutralino-quark scattering.

In the non-relativistic regime, our full calculation is then matched to the spin-independent and spin-dependent operators $Q_{1,2}$ in the effective Lagrangian

$$
\mathscr{L}_{\text {eff }}=c_{1} Q_{1}+c_{2} Q_{2}=c_{1} \bar{\chi} \chi \bar{q} q+c_{2} \bar{\chi} \gamma_{\mu} \gamma_{5} \chi \bar{q} \gamma^{\mu} \gamma_{5} q
$$

as shown symbolically in Fig. 2. As expected, the tree-level coefficients, obtained after a Fierz transformation for the squark processes, agree with those in DarkSUSY [5]. After the one-loop

\footnotetext{
${ }^{2}$ This need not be the case as discussed in Refs. [9, 10].
} 
corrections in the effective theory have also been computed, the matching condition

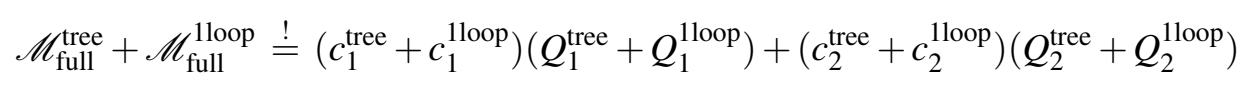

leads to a refactorisation and UV-finite, but scale-dependent redefinitions of Wilson coefficients and operators. In the spin-idependent case, the quark masses $m_{q}(\mu)$ are factorised in $c_{1}$ and run from the high SUSY-breaking scale $1 \mathrm{TeV}$ to the low scale $5 \mathrm{GeV}$, where the nuclear matrix elements are defined. In the spin-dependent case, the running of $c_{2}$ is given by

$$
\frac{c_{2}\left(\mu_{\text {low }}\right)}{c_{2}\left(\mu_{\text {high }}\right)}=\exp \left(\frac{2 n_{f}\left(\alpha_{s}\left(\mu_{\text {high }}\right)-\alpha_{s}\left(\mu_{\text {low }}\right)\right)}{\beta_{0} \pi}\right) .
$$

\section{Numerical results}

Phenomenological minimal SUSY Standard Model (pMSSM) scenarios with eleven free parameters and bino-wino, bino-higgsino, or higgsino-bino DM, that satisfy all current experimental constraints, have been presented in Ref. [2]. Scenario B, e.g., contains a bino-higgsino DM candidate of about $267 \mathrm{GeV}$ mass and up- and down-type squarks of mass 550 and $556 \mathrm{GeV}$, respectively. Fig. 3 shows a scan in the bino mass parameter $M_{1}$ around this point, indicated there by full verti-

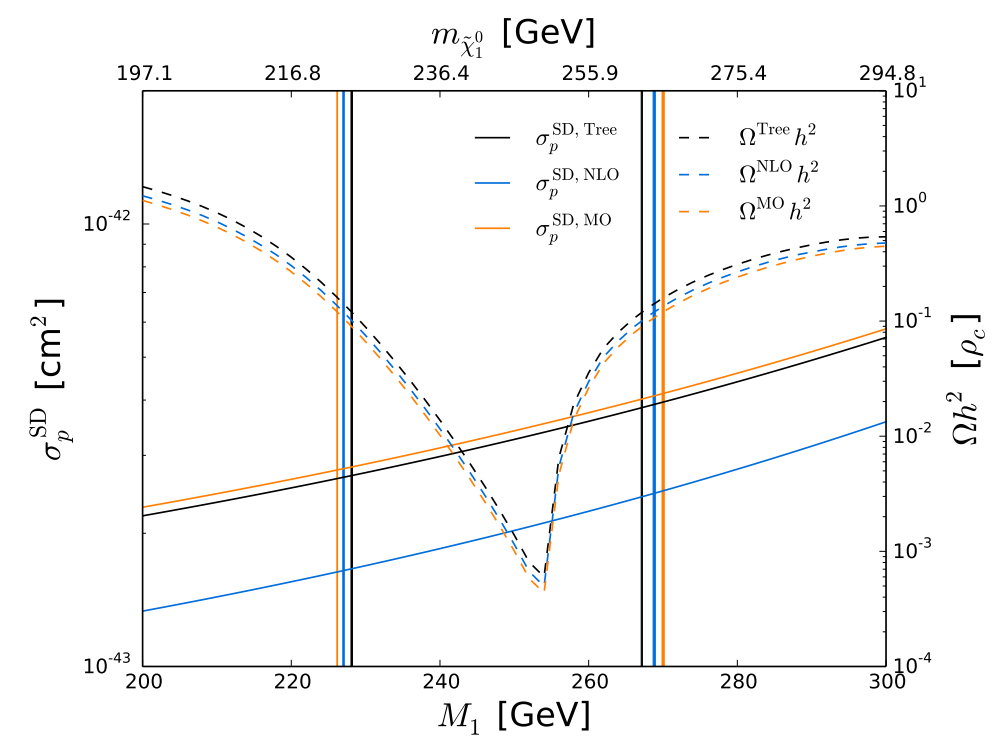

Figure 3: Combined relic density and direct detection calculation in scnenario B.

cal lines at tree-level (black), with micrOMEGAs (orange), and in NLO (blue). In the shown mass region, a second viable scenario with a lower DM mass of about $228 \mathrm{GeV}$ is found, indicated by a second set of vertical lines. One observes good agreement at leading order, but a significant shift at NLO in the spin-dependent direct detection cross section (left ordinate and full curves). The corresponding numbers are listed in Tab. 1. When correlated with the relic density calculations at the same order (dashed curves), this leads to shifts in the extracted bino mass parameter $M_{1}$ of several GeV. In other SUSY scenarios, these effects can even be considerably larger. 
Table 1: Resulting $M_{1}$ and spin-dependent neutralino-proton cross section when combining direct detection and relic density routines in scenario B.

\begin{tabular}{|c|ccc|}
\hline & $M_{1}[\mathrm{GeV}]$ & $\sigma_{p}^{\mathrm{SD}}\left[10^{-43} \mathrm{~cm}^{2}\right]$ & Shift of $\sigma_{p}^{\mathrm{SD}}$ \\
\hline micrOMEGAs & 226 & 2.78 & $+3 \%$ \\
Tree level & 228 & 2.70 & \\
Full NLO & 227 & 1.65 & $-39 \%$ \\
\hline micrOMEGAs & 270 & 4.14 & $+8 \%$ \\
Tree level & 267 & 3.84 & \\
Full NLO & 269 & 2.47 & $-36 \%$ \\
\hline
\end{tabular}

\section{Conclusion}

In conclusion, we have summarised our recent analytical calculation of NLO SUSY-QCD corrections to spin-independent and spin-dependent neutralino-nucleon cross sections, emphasising our choice of renormalisation scheme, the matching of the full diagrammatic calculation to the effective scalar and axial-vector operators, and the renormalisation group running of the Wilson coefficients. More technical issues like our specific tensor reduction method, that avoids vanishing Gram determinants at non-relativistic velocities, were omitted from our discussion, but can be found in Ref. [4].

Numerical results for the direct detection of SUSY DM can now be obtained with DM@NLO for any neutralino decomposition (bino, wino, or higgsino). For a specific bino-higgsino benchmark scenario we found sizeable NLO corrections, which are in fact comparable to the nuclear uncertainties, and we demonstrated that correlations of the relic density and direct detection rates at NLO lead to more precise determinations of the underlying SUSY model parameters.

\section{References}

[1] P. A. R. Ade et al. [Planck Collaboration], Astron. Astrophys. 594 (2016) A13.

[2] J. Harz, B. Herrmann, M. Klasen, K. Kovarik and P. Steppeler, Phys. Rev. D 93 (2016) 114023; see also Phys. Rev. D 91 (2015) 034012, Phys. Rev. D 91 (2015) 034028, Phys. Rev. D 89 (2014) 114012, Phys. Rev. D 87 (2013) 054031, Phys. Rev. D 80 (2009) 085025, and Phys. Rev. D 79 (2009) 061701.

[3] E. Aprile et al. [XENON Collaboration], arXiv:1705.06655 [astro-ph.CO].

[4] M. Klasen, K. Kovarik and P. Steppeler, Phys. Rev. D 94 (2016) 095002; P. Steppeler, PhD thesis, WWU Münster, 2016.

[5] P. Gondolo, J. Edsjo, P. Ullio, L. Bergstrom, M. Schelke and E. A. Baltz, JCAP 0407 (2004) 008.

[6] G. Bélanger, F. Boudjema, A. Pukhov and A. Semenov, Comput. Phys. Commun. 177 (2007) 894.

[7] A. Crivellin, M. Hoferichter and M. Procura, Phys. Rev. D 89 (2014) 054021.

[8] M. Drees and M. M. Nojiri, Phys. Rev. D 47 (1993) 4226.

[9] D. de Florian, R. Sassot, M. Stratmann and W. Vogelsang, Phys. Rev. Lett. 113 (2014) 012001.

[10] J. Li and A. W. Thomas, Nucl. Phys. B 906 (2016) 60.

[11] W. Porod and F. Staub, Comput. Phys. Commun. 183 (2012) 2458. 